\title{
Integrated Combined Amplifiers for Planar Circuits
}

\author{
(Invited paper) \\ Kimberley W. Eccleston \\ Dept of Electrical and Computer Eng.University of Canterbury \\ Christchurch, New Zealand \\ E-mail: kim.eccleston@elec.canterbury.ac.nz
}

\begin{abstract}
Integrating amplification with power combining allows components to be eliminated thereby reducing system size. Recently there has been much interest in reducing size of planar circuit components that use transmission lines, and therefore, combining strategies that employ transmission elements can leverage on these miniaturization techniques to further reduce system size. In this paper we explore the dual-fed distributed power amplifier and a variant that uses periodic loops of transmission lines.
\end{abstract}

\section{INTRODUCTION}

An important application of N-way power dividers is combining $\mathrm{N}$ identical amplifiers to achieve an output power $\mathrm{N}$ times that of a single transistor. Fig. 1 shows the basic schematic of an N-way combining power amplifier. Two Nway combiners are required: one to equally distribute the input signal amongst the $\mathrm{N}$ transistors and another to combine the signals from the transistor outputs. The transverse width of the amplifier is $W$, and the longitudinal lengths of the divider, transistors and combiner are $\mathrm{L}_{d}, \mathrm{~L}_{t}$ and $\mathrm{L}_{\mathrm{c}}$ respectively. Lengths $L_{d}$ and $L_{c}$ include lengths of transmission lines between the transistors and the two dividers to ensure equal phasing of the transistors. The transverse width $W$ is proportional to $\mathrm{N}$.

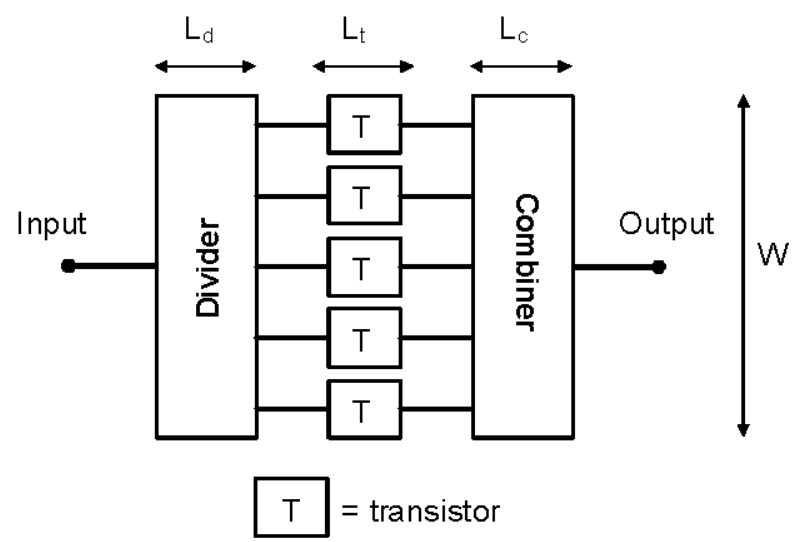

Fig. 1. N-way combined power amplifier block diagram
Experience has shown that corporate power combiners constructed from 2-way dividers [1] are most suitable for the combined amplifier application as they have their output ports located in a straight line. Such dividers however have lengths proportional to the logarithm of $\mathrm{N}$. Tapered line and sectoral power dividers can be used, but like the corporate power divider, their lengths increases with $\mathrm{N}$.

It is common to integrate circuits and components on the same substrate. But as each circuit or component is designed in isolation with little regard to their end application, integration often simply comprises physical placement of components on the same substrate. Though, for on-chip power combining for high-power FETs and power amplifier output stages, dividers are designed to perform impedance transformation [2]. On the other hand, the integrated active antenna concept aims to do more than simply place circuits and components on the same substrate; planar antennas are designed to perform transistor output matching and harmonic termination as well as radiation [3]. A single antenna can also be used to combine transistors [4]-[7].

It is the purpose of this work to consider planar power divider structures that form an integral part of the amplifier (and of course can be integrated on the same substrate). This approach means that individually designed N-way power dividers can be dispensed with thereby reducing circuit size. Such dividers would ideally have longitudinal lengths that are small and independent of $\mathrm{N}$. Whereas it is reasonable to accept that the combined amplifier transverse width, $W$, will be proportional to $\mathrm{N}$. The distributed amplifier is an example of an N-way combined amplifier that has these structural features. However, although conventional distributed amplifiers achieve broad bandwidth, they have very low efficiency. Tapering of the input and output lines of the distributed amplifier can be used to direct transistor output power to the load [8], but the level of combining $(N)$ is limited.

The extended resonance amplifier [9], the dual-fed distributed amplifier [10], and the distributed active transformer [11] offer alternative approaches to efficient integrated combined amplifiers. The former two methods have a longitudinal length largely independent of $\mathrm{N}$, whereas the later method uses an entirely different approach to feed the transistors. The dual-fed distributed amplifier integrates FETs 
in much the same manner that conventional distributed amplifier and their uniform divider and combiner structures lend themselves to method further miniaturization. It is the dual-fed distributed amplifier that is central to this paper.

Section II will describe the basic principles of the dual-fed distributed amplifier, section III describes dual-fed distributed amplifier realizations including modifications to achieve further improve performance and compactness. Section IV will introduce a variant of the dual-fed distributed amplifier that uses periodic loop structure in place of transmission lines.

\section{DUAL-FED DistriBUted AMPLIFIER PRINCIPLES}

The dual-fed distributed amplifier was originally proposed as far back as 1988 as an approach to make better use of the transistors [12]. More recent activity concentrated on efficient power combining and utilization of the transistors [10][13]. A basic schematic of an optimum 4-FET dual-fed distributed amplifier is shown in Fig. 2.

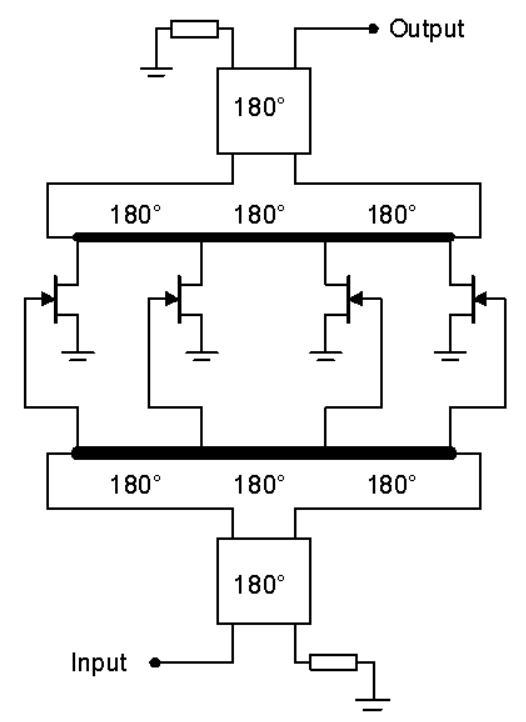

Fig. 2. Dual-fed distributed amplifier combining 4 FETs

The midpoint of the gate and drain transmission line are effectively short-circuited to ground and allows the dual-fed distributed amplifier to be split into a pair of single-ended dual-fed distributed amplifiers which can be combined using a pair of quadrature couplers to achieve matched input and output ports [14][15]. The single-ended dual-fed distributed amplifier can be designed for efficient operation [15], and an $\mathrm{N}$-way single-ended dual-fed distributed amplifier is shown in Fig. 3. Dual-feeding in this amplifier occurs by virtue of the short-circuit terminations of both the gate and drain lines which are also useful for FET biasing.

An important attribute of the dual-fed distributed amplifiers shown in Fig. 2 and Fig. 3 is that all transistors are equally driven, drive into identical loads, and are equally utilized. Dual-feeding also provides some compensation for losses in the gate and drain lines [13]. With reference to Fig. 3, it can be shown that the load impedance for each FET is zero at even harmonics, and $N Z_{L}$ at the fundamental and odd harmonics [16]. The drain line characteristic impedance $Z_{0 \mathrm{D}}$ is normally equal to $Z_{\mathrm{L}}$ (at the fundamental) to maximize bandwidth.

\section{DUAL-FED Dis TRIBUTED AMPLIFIER REALIZATIONS}

A balanced amplifier comprising a pair optimum 2-FET single-ended dual-fed distributed amplifier was designed for operation at $1.8 \mathrm{GHz}$ and achieved an efficiency of $37 \%$ under class-A conditions [17] and $50 \%$ under class-B conditions [18]. In both cases, the output power was $25 \mathrm{dBm}$, and the gain was $9.3 \mathrm{~dB}$ and $7.3 \mathrm{~dB}$ under class-A and class-B conditions respectively [18]. Class-B operation is possible due to the inherent short-circuit loading of the FETs at even harmonics [18]. This amplifier had matched input and output ports.

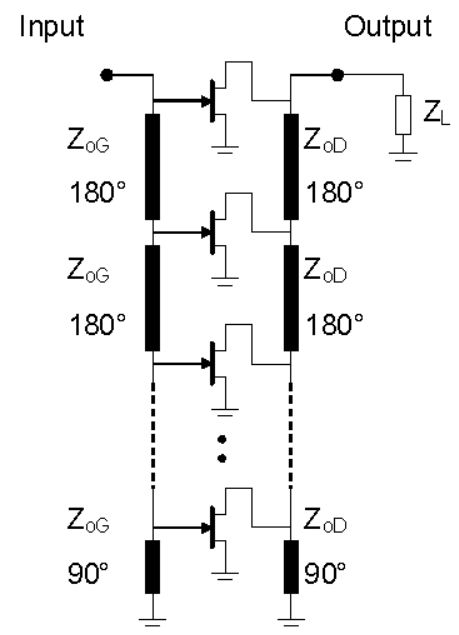

Fig. 3. N-way single-ended dual-fed distributed amplifier.

Class-F operation of FETs is possible at microwave frequencies and requires the presence of odd-harmonics [19]. The single-ended dual-fed distributed amplifier can be modified to achieve the right conditions under which class-F operation can take place and yet no harmonics appear at the output. Namely, $Z_{\mathrm{L}}$ is resistive at the fundamental and infinite at odd-harmonics, and that the output matching network contains odd-harmonic traps [16]. A 2-FET class-F singleended distributed amplifier operating at $1.8 \mathrm{GHz}$ achieved an efficiency of over $70 \%$ at an output power of $22 \mathrm{dBm}$ and had a gain of $8 \mathrm{~dB}[20]$.

The transmission line lengths between FETs are nominally $180^{\circ}$ and the gate and drain lines are terminated by $90^{\circ}$ shortcircuit stubs. FET input and output capacitances mean that these line lengths need to be shortened [21] to maintain correct phasing of the FETs [17]. Although shortening of the transmission lines is desirable, bandwidth is also reduced [22]. An alternative approach is to implement the gate and drain 
lines with artificial transmission lines. Using this approach, not only is the transverse width, $\mathrm{W}$, reduced, the FET input and output capacitances are absorbed into the artificial transmission line structure without reducing bandwidth [22]. Fig. 4 shows the photograph of 3-FET single-ended dual-fed distributed amplifier that operates at $1.8 \mathrm{GHz}$. This amplifier achieved and output power of $23 \mathrm{dBm}$ over an $11 \%$ bandwidth and over $35 \%$ efficiency over a $15 \%$ bandwidth [22]. Another approach to reduce size is to use the metamaterial approach to realize finite length zero-phase shift transmission lines [23]. This has the added bonus of increasing bandwidth [23].

Recently, optimum single-ended dual-fed distributed amplifiers have been used to implement the main and peaking amplifiers of a Doherty amplifier [24][25].

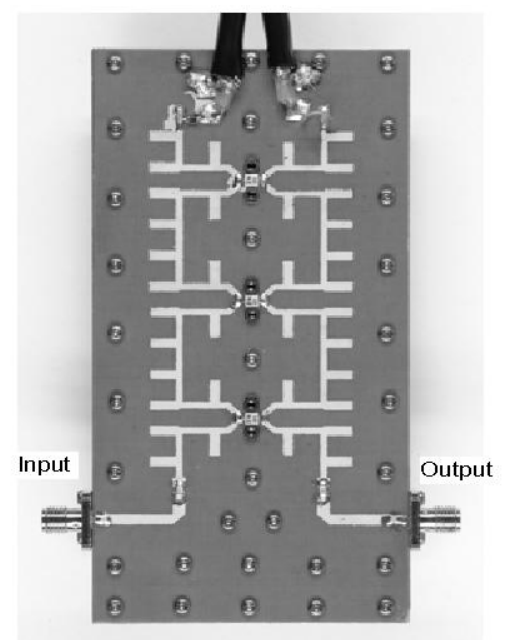

Fig. 4. 3-FET 1.8 GHz single-ended dual-fed distributed amplifier that uses artificial transmission lines [22]

\section{COMBINING of FETs Using PerIodic LoOP Structures}

One of the drawbacks of the optimum dual-fed distributed amplifier is that the load seen by the FET is $\mathrm{N}$ times the amplifier load impedance. For power FETs, a low value of load impedance is required and hence the amplifier load impedance would be prohibitively low. Preferably, a combiner should present the power FET a load impedance that is the amplifier load impedance divided by $\mathrm{N}$. In this way, a low impedance can be obtained from an external $50 \Omega$ amplifier load. Fork power dividers operate on this principle.

Fig. 5 shows the schematic of the proposed new type of dual-fed distributed amplifier that uses a periodic loop combining structure in place of the drain transmission line. The FET gates are fed similar to a pair of 2-FET single-ended dual-fed distributed amplifiers but with a $180^{\circ}$ hybrid feeding these. In the periodic loop combining structure, the characteristic impedance of all the transmission line elements are identical and equal to $Z_{0 \mathrm{C}}$. Simulations have shown that the load impedance presented to each FET are all equal to $Z_{0 C}{ }^{2} / N Z_{L}$ where $Z_{\mathrm{L}}$ is the amplifier load impedance.
Importantly, the FET load impedance is inversely proportional to $\mathrm{N}$, and similar to the dual-fed distributed amplifier, the longitudinal length of the combiner is independent of $\mathrm{N}$.

An amplifier operating at $2 \mathrm{GHz}$ employing four Eudyna FLC057WG GaAs FETs is now considered. For such a FET, the optimum load is $33 \Omega$ when a $3.6 \mathrm{~V}$ drain supply is used. If $Z_{\mathrm{L}}$ is $50 \Omega$, then $Z_{0 C}=81 \Omega$ which is feasible. Shunt inductors were used to parallel resonate the FET input and output at 2 $\mathrm{GHz}$.

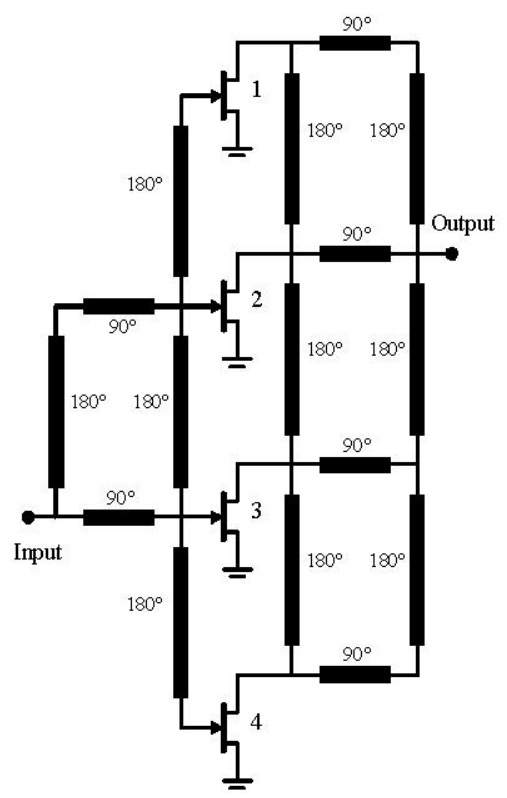

Fig. 5. Schematic of a 4-FET amplifier using a periodic loop combiner at the output of the amplifier

Harmonic balance simulations were used to assess the feasibility of the amplifier. The FETs were represented by a nonlinear circuit model and included parasitics, whose parameters were obtained using the data sheet DC I/V data and S-parameters.

Simulated load lines indicated that the load impedances seen by the FETs at $2 \mathrm{GHz}$ were identical and optimum for class-A operation. Fig. 6 shows the loadlines at $1.85 \mathrm{GHz}$.

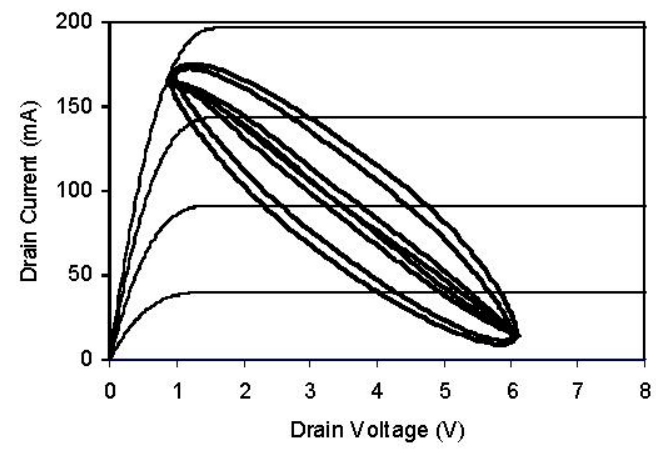

Fig. 6. Simulated loadlines for the 4-FET power amplifier at $1.85 \mathrm{GHz}$

Fig. 7 shows the simulated transfer characteristics at $2 \mathrm{GHz}$ as a function of input power. The output power and efficiency 
at compression are consistent with theoretical expectations for the FETs operated from a $3.6 \mathrm{~V}$ supply voltage. Other simulations showed that the gain is flat over $500 \mathrm{MHz}$ or $25 \%$ bandwidth and even harmonic suppression is obtained.

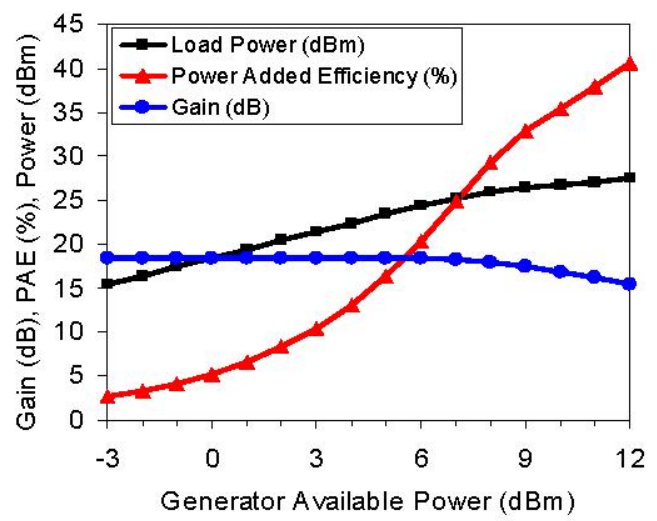

Fig. 7. Simulated large-signal transfer characteristics as a function of input generator power for the 4-FET power amplifier at $2 \mathrm{GHz}$

The input power divider and output combiner comprise transmission lines and artificial transmission line methods can be used to reduce size, fill the space of loops, and absorb FET input and output capacitances [22].

\section{CONCLUSION}

In this paper we have examined combined power amplifiers that integrate the input divider and output combiner functions as part of the amplifier circuit thereby obviating the need for extra power dividers and interconnecting lines. These amplifiers were the: (i) dual-fed distributed amplifier, and (ii) a new type of amplifier that comprises a periodic loop combining structure. Both amplifiers have a longitudinal length independent of $\mathrm{N}$ the number of transistors to be combined. Their composition makes them amenable to miniaturization using artificial transmission line methods which can be used to absorb transistor input and output capacitances into the divider and combiner structures. In both cases, even harmonic suppression occurs. The load presented to the transistors is proportional to $\mathrm{N}$ for the dual-fed distributed amplifier, and inversely proportional to $\mathrm{N}$ for the proposed amplifier. The former amplifier is evolved whist further investigations are needed for the later amplifier.

\section{REFERENCES}

[1] J. Zhou, K. A Morris and M. J. Lancaster, "General Design of Multiway Multisection Power Dividers by Interconnecting Two-Way Dividers", IEEE Trans. Microwave Theory and Tech., vol. 55, no. 10, pp 2208 2215 , Oct. 2007.

[2] M. Mochizuki, Y. Itoh, M. Kohno, H. Masuno and T. Takagi, "C-X Band 14W Power Amplifier Having Flat Gain and Power Response", 1993 IEEE Int. Microwave Symp., MTT-S, pp 1365 - 1368.

[3] K. Chang, R. A. York, P. S. Hall and T. Itoh "Active Integrated Antennas," IEEE Trans. Microwave Theory and Tech., vol. 50, no. 3, pp 937 - 944, March 2002.
[4] W. R. Deal, V. Radisic, Y. Qian and T. Itoh, "Novel Push-Pull Integrated Antenna Transmitter Front-End", IEEE Microwave and Guided Wave Letters, vol. 8, no. 11, pp 405 - 407, Nov. 1998.

[5] S. Gao and P. Gardiner, "Integrated Antenna/Power Combiner for LINC Radio Transmitters", IEEE Trans. Microwave Theory and Tech., vol. 53, no. 3, pp 1083 - 1088, March 2005.

[6] K. W. Eccleston, "Four-Way Power Combining Integrated Antenna", 2006 Asia Pacific Microwave Conference (APMC2006), Yokohama, Japan, 12 - 15 December 2006, pp 828 -831.

[7] L. Chiu, T. Y. Yum, C. H. K. Chin, Q. Xue and C. H. Chan, "Highefficiency class-B push-pull amplifying array for microwave transmitting front end", IEE Proceedings on Microwaves, Antennas and Propagation, vol. 153 , no. 1 , pp $25-28$, Feb. 2006.

[8] M. Ross, R. G. Harrison and R. K. Surridge, "Taper and Forward-Feed in GaAs MMIC Distributed Amplifiers", 1989 IEEE Int. Microwave Symp., MTT-S, pp 1039 - 1042.

[9] A. L. Martin, A. Mortazawi and B. C de Loach, "An Eight-Device Extended-Resonance Power-combining Amplifier", IEEE Trans. Microwave Theory and Tech., vol. 46, no. 6, pp 844 - 850, June 1998.

[10] K. W. Eccleston, "Design considerations for the dual-fed distributed power amplifier", 2000 Asia Pacific Microwave Conference (APMC 2000), 3 - 6 Dec 2000, Sydney, Australia, pp 205 - 208.

[11] I. Aoki, S. D. Kee, D. B Rutledge and A. Hajimiri, "Distributed Active Transformer - A New Power Combining and Impedance-Transformation Technique", IEEE Trans. Microwave Theory and Tech., vol. 50, no. 1, pp 316 - 331, Jan. 2002.

[12] C. S. Aitchison, N Bukhari, C. Law and N. Nazoa-Ruiz, "The Dual-Fed Distributed Amplifier", 1988 IEEE Int. Microwave Symp., MTT-S, pp $911-914$

[13] K. W. Eccleston, "Output Power Performance of Dual-Fed and SingleFed Distributed Amplifiers", Microwave and Opt. Tech. Letters, vol. 27, no. 4 , pp $281-284.20$ Nov 2000.

[14] M. R. Moazzam and C. S. Aitchison, "A high gain dual-fed single stage distributed amplifier," 1994 IEEE Int. Microwave Symp., MTT-S, pp. 1409-1412.

[15] K. W. Eccleston, "Design and performance of a balanced single-ended dual-fed distributed power amplifier", 2001 Asia-Pacific Microwave Conference, 3-6 December 2001, National Taiwan University, Taipei, Taiwan, pp 1187-1190.

[16] K. W. Eccleston, "Design of a Class-F Single-ended Dual-fed Distributed Amplifier", 2003 Asia Pacific Microwave Conference (APMC2003), 4-7 November 2003, Seoul, South Korea, pp 653-656.

[17] K. W. Eccleston and O. Kyaw, "FET Power Combining Using the Balanced Single-Ended Dual-Fed Distributed Amplifier Approach", Microwave and Opt. Tech. Letters, vol 35, no. 1, pp 42-45, 5 October 2002.

[18] K. W. Eccleston and O. Kyaw, "Analysis and Design of Class-B DualFed Distributed Power Amplifiers", IEE Proceedings. H, Microwaves, Antennas and Propagation, Vol. 151, No. 2, April 2004, pp 104-108.

[19] P. Colantonio, F. Giannini, G. Leuzzi, and E. Limiti, "On the class-F power amplifier design," Int. J. RF Microwave CAE, vol. 9, no. 2, pp. 129-149, Mar. 1999.

[20] K. W. Eccleston, "Modified Class-F Distributed Amplifier", IEEE Microwave and Wireless Component Letters, Vol. 14, No. 10, October 2004, pp 481- 483.

[21] T. Hirota, A. Minakawa and M. Muraguchi, "Reduced-size branch-line and rat-race hybrids for uniplanar MMICs", IEEE Trans. Microwave Theory and Tech., vol. 38, no. 3, pp 270 - 275, March 1990.

[22] K. W. Eccleston, "Compact Efficient Dual-Fed Distributed Power Amplifier", IEEE Trans. Microwave Theory and Tech., vol. 53, no. 3, March 2005, pp 825 - 831 .

[23] K. W. Eccleston, "Application of Left-Handed Media in Distributed Amplifiers", Microwave and Opt. Tech.Letters, vol. 44, no. 6, pp 527 533, 20 March 2005.

[24] K. J. Cho, W. J. Kim, S. P. Stapleton, J. H. Kim, B. Lee, J. C. Choi, J. Y. Kim and J. C. Lee, "Design of N-way distributed Doherty amplifier for WCDMA and OFDM applications", IET Electronics Letters, vol. 43, no. 10, pp 577-579, 10 May 2007.

[25] K. J. Cho, W. J. Kim, J. Y. Kim, J. H. Kim and S. P. Stapleton, "A novel N-way distributed Doherty amplifier with improved efficiency at high PAR signals", Microwave Journal, vol. 51, no. 4, available online, April 2008. 